\title{
Prevalencia de tumores odontogénicos en el Hospital Base Valdivia, periodo 1989-2008
}

\section{Odontogenic tumors in Valdivia: Prevalence from 1989 to 2008}

\author{
Thiers L C*, Sotomayor C C ${ }^{1 * *}$, Peters F I***, Lantaño P C***, Thiers L S****
}

\section{RESUMEN}

Objetivo: Este artículo corresponde a un estudio observacional de tipo descriptivo de corte transversal, tiene como objetivo determinar la prevalencia de tumores odontogénicos en la población atendida en el Hospital Base Valdivia, en un periodo de 20 años (1989-2008), según la nueva clasificación de lesiones tumorales de la WHO, 2005.

Metodología: Se revisaron 2.078 informes de biopsias correspondientes a lesiones de la cavidad oral de los archivos de informes del servicio de Anatomía Patológica, Hospital Base Valdivia entre enero del año 1989 y diciembre del año 2008. Como criterios de inclusión están la presencia de un diagnóstico histopatológico en la ficha y legibilidad de ésta. Las variables a analizar incluyen: edad, género, diagnóstico histopatológico, tipo de tumor y área de localización. Los datos recolectados fueron tabulados en planilla de base de datos, para su posterior análisis estadístico.

Resultados: De las 2.078 biopsias del territorio bucal, 31 corresponden a tumores odontogénicos, lo que representa un $1,5 \%$ de todas las lesiones biopsiadas del territorio oral. Una lesión maligna fue encontrada y corresponde a carcinoma ameloblástico infiltrante. La lesión más frecuente correspondió a tumor odontogénico queratoquístico (41,9\%), seguido por el ameloblastoma $(22,6 \%)$ y, por último, odontoma $(16,1 \%)$. El promedio de edad de 35,7 años. El área más frecuente de presentación es el área molar mandibular (54,8\%). Conclusión: Los tumores odontogénicos son de baja prevalencia en la población valdiviana atendida en el Hosptial Base Valdivia entre los años 1989-2008. No tiene predilección por género. La lesión más prevalente es el tumor odontogénico queratoqísitico.

Palabras clave: Tumores odontogénicos, prevalencia tumores, quistes maxilares.

\section{SUMMARY}

Objetive: This article corresponds to a descriptive cross sectional study, which aims to determine the prevalence of primary odontogenic tumors in population attending Hospital Base Valdivia, during a period of 20 years (1989-2008), based on the WHO's 2005 new classification of tumors.

Methodology: 2,078 reports of biopsies for lesions oral cavity from Pathology Service Hospital Valdivia Base, between January 1989 and December 2008 were reviewed. The presence of an histologically confirmed diagnosis in the patient's chart and readability of it were considered as inclusion criteria. The variables analyzed

\footnotetext{
1 Instituto de Salud Pública. Facultad de Medicina. Universidad Austral de Chile.

* $\quad$ Cirujano Dentista.

** Médico Veterinario, Mg.

*** Cirujano Dentista.

**** Estudiante de Odontología. Universidad Austral de Chile.
} 
included age, gender, histological diagnosis tumor type and location of this area. The collected data was tabulated in database spreadsheet for subsequent statistical analysis.

Results: Of the 2,078 biopsies of oral territory, 31 correspond to tumors odontogenic, representing $1.5 \%$ of all biopsied lesions territory oral. A malignant lesion was found and corresponds to ameloblastic carcinoma infiltrating. The most frequent lesion corresponded to odontogenic keratocyst tumours (41.9\%), followed by ameloblastoma (22.6\%), and finally odontoma (16.1\%). The average presentation age is 35.7 years. The most common area of presentation is the mandibular molar area (54.8\%).

Conclusion: Odontogenic tumors are of low prevalence in Hospital Base Valdivia between 1989 to 2008. It's more often in men, no gender predilection, being the most prevalent lesion tumor odontogenic keratocysts.

Key words: Odontogenic tumors, tumors prevalence, maxillary cysts.

Fecha de recepción: 27 de julio de 2012.

Aceptado para publicación: 19 de septiembre de 2012.

Thiers L C, Sotomayor C C, Peters F I, Lantaño P C, Thiers L S. Prevalencia de tumores odontogénicos en el Hospital Base Valdivia, periodo 1989-2008. Av. Odontoestomatol 2013; 29 (6): 303-308.

\section{INTRODUCCIÓN}

Los tumores odontogénicos (TO) se forman a partir del epitelio o ectomesénquima, implicados en la formación de los dientes (1). Son entidades agresivas localmente y sus tratamientos difieren bastante entre sí. Su frecuencia de presentación varía según región geográfica, etnia y género entre otras.

Los TO son lesiones de baja presentación en la población mundial en contraste con otros tipos de lesiones tumorales que afectan a los maxilares, como son los tumores en glándulas salivales, cuya prevalencia alcanza un 10,5\%; el carcinoma de células escamosas con un $8,3 \%$ y quistes de los maxilares con una frecuencia cercana al $7 \%$ (2).

Estudios brasileños indican que los tumores odontogénicos corresponderían al 1,8\% del total de las lesiones orales y tumorales que afectan a los maxilares (3).

Estos tumores son lesiones exclusivas de los maxilares. Engloban un gran número de entidades patológicas que se han dividido, según sus características clínicas e histológicas en neoplasias benignas, malignas y hamartomas.

El año 2005, la WHO divulgó la última modificación de la clasificación de patologías tumorales, en don- de algunas lesiones fueron introducidas y otras cambiadas de categoría (2), comparativamente a la ya existente y actualmente obsoleta del año 1992. Es el caso del queratoquiste, antiguamente considerado un quiste odontológico del desarrollo, el cual cambió su clasificación a tumor odontogénico queratoquístico (TOQ) debido a su comportamiento invasivo, potencial agresivo y naturaleza neoplásica.

En Chile, Ochsenius et al. (2002) evaluaron archivos de biopsias entre 1975 y 2000 del Instituto de Referencia de Patología Oral de la Universidad de Chile (IREPO); las placas de hematoxilina-eosina fueron re-revisadas por dos observadores para su posterior reclasificación según la edición de la WHO del 1992; se determinó una frecuencia de tumores odontogénicos de un $1,3 \%$, en relación a todas las lesiones que afectan a la cavidad oral y maxilares, liderando la frecuencia de presentación el odontoma $(44,7 \%)$, seguido por el ameloblastoma $(20,6 \%)$ y por último el mixoma odontogénico $(8,9 \%)$.

En cuanto a malignidad de las lesiones, existe una predominancia de lesiones benignas sobre las malignas siendo asintomáticos en la mayoría de los casos (5).

En cuanto a la edad promedio general de presentación de tumores benignos, esta alcanza los 25,2 años, 
específicamente de 24,5 años para género masculino y hombres y 25,8 años para el femenino (4).

Debido a la ausencia de bases de datos en la Región de los Ríos, y a que estos TO son diagnósticos diferenciales entre las múltiples patologías que afectan a los maxilares, el objetivo de este estudio es determinar la prevalencia de tumores odontogénicos en la población atendida en el Hospital Base Valdivia, en un periodo de 20 años (1989-2008) en base a la nueva clasificación de lesiones tumorales de la WHO, 2005.

\section{MATERIAL Y MÉTODO}

La investigación corresponde a un estudio descriptivo de corte transversal censal, cuya población en estudio consideró a todos aquellos pacientes que presentaran lesiones en el territorio bucal con envío a biopsia en el periodo comprendido entre enero 1989 y diciembre 2008. Como criterios de inclusión se consideró la presencia de un diagnóstico histopatológico en la ficha, además de la respectiva legibilidad de ésta. Las variables a analizar incluyen edad; género; diagnóstico específico según histopatología; tipo de tumor (benigno, maligno) y área de localización de este (se clasificó para ambos maxilares en

\begin{tabular}{|l|r|r|c|c|c|c|}
\hline \multicolumn{7}{|c|}{ TABLA 1.- DISTRIBUCIÓN DE TUMORES } \\
ODONTOGÉNICOS SEGÚN GÉNERO. \\
HOSPITAL REGIONAL BASE VALDIVIA, \\
\hline \multirow{7}{*}{ Tumor } & \multicolumn{2}{|c|}{ Total } & \multicolumn{2}{c|}{ Masculino } & \multicolumn{2}{c|}{ Femenino } \\
\cline { 2 - 7 } & $\mathrm{N}$ & $\%$ & $\mathrm{~N}$ & $\%$ & $\mathrm{~N}$ & $\%$ \\
\hline $\begin{array}{l}\text { Benignos } \\
\text { - TOQ }\end{array}$ & 13 & 41,9 & 7 & 53,9 & 6 & 46,2 \\
- AMB & 7 & 22,6 & 4 & 57,1 & 3 & 42,9 \\
- OD & 5 & 16,1 & 2 & 40 & 3 & 60 \\
- TOA & 3 & 9,7 & & & 3 & 100 \\
- MO & 1 & 3,2 & & & 1 & 100 \\
- OA & 1 & 3,2 & 1 & 100 & & \\
Malignos & & & & & & \\
- Ca. AMB & 1 & 3,2 & 1 & 100 & & \\
\hline Total & 31 & 100 & 15 & 48,3 & 16 & 51,6 \\
\hline
\end{tabular}

área anterior, premolar y molar; en el caso del área molar mandibular, incluye rama ascendente).

Se revisaron 2.078 informes de biopsias correspondientes a lesiones de la cavidad oral de los archivos de informes del Servicio de Anatomía Patológica, Hospital Base Valdivia entre enero del año 1989 y diciembre del año 2008. Se extrajeron los datos correspondientes a los parámetros considerados anteriormente dentro de las lesiones del territorio oral. Con respecto a los casos recurrentes, con más de una biopsia, se consideró como único caso al diagnóstico histopatológico definitivo.

La identificación de los tumores está basada en la nueva clasificación del año 2005 de la WHO. Para esto, los casos de queratoquiste fueron reanalizados y recategorizados según el tipo de lesión que correspondiese. Los datos recolectados fueron tabulados en planilla de base de datos para su posterior análisis estadístico mediante el programa estadístico PASW STATISTIC 18. Se consideró la confección de tablas resumen y gráficas de acuerdo a los parámetros analizados; determinación de frecuencias absolutas y relativas de las variables en estudio y cálculo de la media.

\section{RESULTADOS}

De las 2.078 biopsias del territorio bucal, el 1,5\% corresponde a tumores odontogénicos, lo que equivale

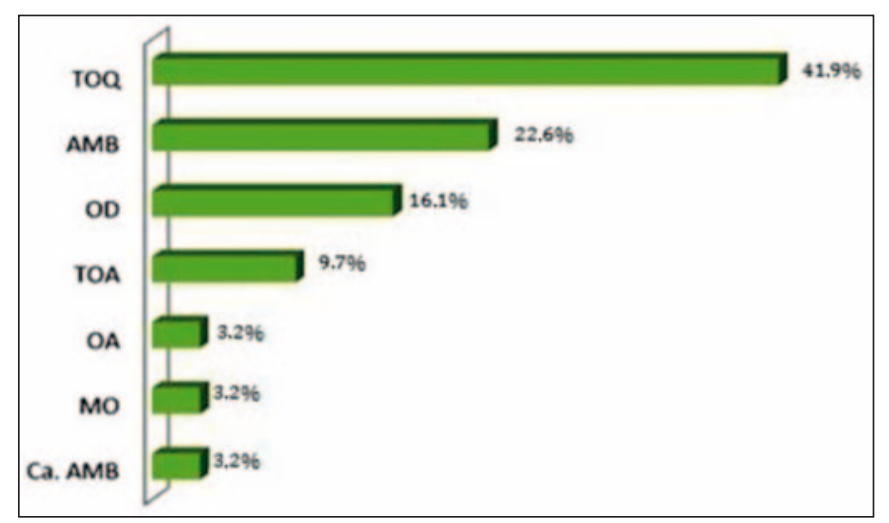

Fig. 1. Prevalencia de TO según el tipo de tumor. Hospital Base Valdivia. 1989-2008.

TOQ: Tumor odontogénico queratoquístico; AMB: Ameloblastoma; OD: Odontoma; TOA: Tumor odontogénico adenomatoide; OA: Odontoameloblastoma; MO: Mixoma odontogénico; Ca. AMB: Carcinoma ameloblástico. 
a 31 lesiones tumorales. De los 31 tumores, 30 corresponden a lesiones benignas. La lesión maligna encontrada, corresponde a carcinoma ameloblástico infiltrante, en un paciente de sexo masculino de 39 años de edad. En relación a los tumores benignos, el mayor porcentaje recayó en el tumor odontogénico queratoquístico $(41,9 \%)$, seguido por ameloblastoma $(22,6 \%)$ y odontomas odontogénicos $(16,1 \%)$.

De todos los tumores diagnosticados, 15 lesiones se presentaron en hombres (48,3\%), incluyendo la lesión maligna, respecto a 16 en mujeres. El índice de presentación de lesiones benignas según género masculino: femenino es de 1:1,3.

El rango de edad de los pacientes afectados considera el intervalo entre los 5 y 75 años, con un promedio general de edad de 35,7 años. Un 74,1\% de las lesiones se concentra en las primeras 6 décadas de vida, con un peak de presentación en la segunda década. El promedio de edad para hombres, sin diferenciación según malignidad de la lesión, es de 37,6 años, con un incremento en la segunda década. La edad promedio para las mujeres es de 33 años.

Según zona anatómica de presentación de lesiones benignas, la más frecuente corresponde a la zona molar mandibular $(54,8 \%)$, seguida por la zona anterior maxilar (16,1\%). La lesión tumoral maligna encontrada se presentó abarcando el hemimaxilar derecho, teniendo como límite posterior la zona de molares.

La lesión más frecuente corresponde al tumor odontogénico queratoquístico $(41,9 \%)$, presentándose mayormente en hombres (53,9\%); sin embargo, no existe predilección por sexo ( $p>0,05)$. El rango etario incluye desde los 16 a los 72 años, siendo el promedio de edad de 43,1 años; un 30\% de las lesiones se presentaron en la $6^{\circ}$ década de vida. La zona anatómica más frecuentemente comprometida corresponde al área molar mandibular (69,2\%); 4 lesiones se encontraron en el maxilar; 2, en la región anterior y 2 , en la región molar.

Ameloblastoma es el segundo tumor odontogénico más frecuente (22,6\%). Se presenta con mayor recurrencia en hombres $(57,1 \%)$. En cuanto a la edad, esta presenta un rango que oscila entre los 14 y 75 años, con un peak en la $2^{\circ}$ década. La zona molar mandibular es el área más comúnmente afectada $(57,1 \%)$. En relación a la variante histológica de ameloblastoma, la forma uniquística se presenta en un $57,1 \%$ de los casos; la sólida, en un $43 \%$.

En tercer lugar, se posiciona el odontoma, con un $16,1 \%$ del total de las lesiones tumorales, afectando mayoritariamente a mujeres (60\%). El rango etario va desde los 5 a los 18 años, con un incremento en la primera década de vida. Se presentó con igual frecuencia en zona anterior maxilar y mandibular (40\%). Con respecto al tipo específico de odontoma, el más frecuente fue el odontoma compuesto (60\%).

\section{DISCUSIÓN}

Según la nueva clasificación de WHO, 2005, se obtuvo una prevalencia de tumores de origen odontogénico de un 1,5\%; el tumor que con mayor frecuencia se presentó fue el tumor odontogénico queratoquístico, en la población atendida en el Hospital Base Valdivia en un periodo de 20 años.

Este estudio de corte trasversal censal recolectó la totalidad de los datos que respecta a biopsias de la cavidad oral de la población en estudio. Se generaron nuevos antecedentes en cuanto a la prevalencia de este tipo de lesiones en la región de Los Ríos, pues no existe algo similar. Sin embargo, se debe señalar, que en cualquier investigación que utiliza diferentes juicios clínicos para realizar diagnósticos, es difícil obtener una confiabilidad universal para la obtención de resultados rigurosos; sería necesario que los diagnósticos fuesen reanalizados y clasificados nuevamente por un profesional calibrado e instruido en cuanto a patología tumoral de la cavidad oral, aumentando la validez externa de la investigación, otorgándole más fuerza a los resultados obtenidos. Además, no todas las lesiones fueron derivadas a biopsia, como es el caso del odontoma. Debe considerarse el sesgo de prevalencia que un estudio basado en datos previamente obtenidos de fichas clínicas e informes de biopsias puede presentar.

La prevalencia encontrada (1,5\%), indica la baja frecuencia de presentación las lesiones tumorales de la cavidad bucal. Avelar et al. (2008) realizó estudios 
similares en Brasil utilizando la nueva clasificación de la WHO 2005, determinando una prevalencia de tumores odontogénicos de un 4,8\%. Sin embargo, Fernandes et al. (2005) arroja una prevalencia de un $1,8 \%$, en base a la antigua clasificación tumoral de la WHO 1992. En cuanto a la prevalencia de lesiones según género, es ligeramente más alta, tal como lo han demostrado Avelar et al. (2008) en Brasil, Luo \& Li (2009) en China, y Tawfik et al. (2010) en Egipto. Es necesario mencionar que Ochsenuis et al. (2002), en Chile, obtuvo una ligera preferencia por el género femenino en sus resultados.

Respecto a la edad, el rango presentado fue de 5 a 75 años con un promedio de edad para los tumores de 35,7 años, indicador más alto comparado con estudio en Brasil de Avelar et al. (2008), donde el promedio alcanza tan solo los 30,7 años. El promedio de edad en lesiones benignas encontradas en Valdivia alcanza los 35,1 años; este hallazgo difiere de lo informado por Ochsenuis et al. (2002), donde el promedio es sustancialmente más bajo, con tan solo 25,2 años. Debe considerarse que Ochsenius et al. (2002) determinó como el tumor más prevalente al Odontoma, caracterizado por una alta prevalencia en pacientes menores de 20 años.

En general, los tumores benignos se presentaron con una marcada preferencia por el hueso mandibular, específicamente en la zona molar. Similar tendencia muestra Ochsenius et al. (2002). Estudios en China realizados por Jing et al. (2007), indican un porcentaje mayor en cuanto a presentación en área molar mandibular. Con aun mayor frecuencia, Tawfik et al. (2010) en Egipto indican presencia de lesiones casi exclusivamente en el área molar mandibular.

La lesión más frecuente encontrada en el presente estudio corresponde al tumor odontogénico queratoquístico $(41,9 \%)$; similares resultados presentan estudios en Brasil, con un 30\% (5), Libia con un $35,1 \%$ (6) y China con un 38,7\% (2). Ochsenius et al. (2002), en Chile, encontró como lesión más prevalente al odontoma, con un $44,7 \%$ de todas las lesiones benignas. Cabe señalar que la clasificación utilizada en el mencionado estudio no corresponde a la utilizada en el presente estudio, puesto que no considera al tumor odontogénico queratoquístico, En las biopsias evaluadas, el tumor odontogénico que- ratoquístico fue más frecuente en género masculino (53,8\%), resultados también encontrados en China (Luo \& Li, 2009) y Libia (6). En Brasil por el contrario, se presentó una ligera predilección por el género femenino (5). Respecto al TOQ según edad, el presente estudio indica un promedio de 43,1 años. Avelar et al. (2008) muestra un promedio etario menor, con 31 años en Brasil; en China Luo $\mathcal{E}$ Li (2009) determinó un promedio de 33 años; similar hallazgo en Libia, donde el $52 \%$ de los pacientes que presentan esta lesión se encuentran entre los 21 y 30 años (6).

El ameloblastoma correspondió a la segunda lesión más común (22,6\%). Similares resultados arrojaron reportes en Brasil (5), con un 23,7\%. En Libia, ElGehani et al. (2009) plantea una prevalencia de un $22,3 \%$. Esta frecuencia es ligeramente más alta comparada con el reporte de un $20,6 \%$ publicado por Ochsenius et al. (2002). El ameloblastoma es considerado en ciertos países de África tales como Egipto el tumor más frecuente. Tawfik et al. (2010) refiere a una prevalencia de un $41,4 \%$, muy superior en contraste con estudios latinoamericanos.

La prevalencia de odontomas $(16,1 \%)$ fue más alta que en reportes de países de África y Asia. Tawfik et al. (2010) y Luo \& Li (2009) reportan un 13,5\% y un $6,1 \%$ respectivamente. En Libia, El-Gehani et al. (2009) menciona un 19\%. Guerrisi, Piloni \& Keszler (2007) muestra una alta prevalencia del Odontoma en estudio realizado en Argentina (50,9\%) en población menor de 20 años. En Brasil, este alcanza un 22,6\% (5). Ochsenius et al. (2002) reporta a nivel nacional una relativa alta prevalencia de odontomas $(44,7 \%)$, en comparación a lo encontrado en el presente reporte. No es claro si estas diferencias en la prevalencia de la lesión se deben a sesgos de prevalencia, a la carga genética asociada al componente racial, o más bien a un factor medio ambiental.

Según las variantes histológicas, la más frecuente fue el odontoma compuesto; resultados similares fueron presentados en estudio argentino (7).

Clínicamente es recomendable tener presente la prevalencia de estas lesiones tumorales en la población, pues son diagnósticos diferenciales entre las muchas patologías que afectan a los maxilares. El 
conocimiento de las características de presentación de los tumores odontogénicos entrega una aproximación diagnóstica previa a la biopsia, examen que confirmará el diagnóstico definitivo de la lesión tumoral.

En conclusión, los tumores odontogénicos son de baja prevalencia en la población atendida en el Hospital Base Valdivia entre los años 1989-2008. Se presentan con mayor frecuencia en género masculino, sin presentar mayor predilección por sexo. La lesión más prevalente es el tumor odontogénico queratoquístico.

Es necesario realizar futuras investigaciones que puedan crear hipótesis en cuanto a la presentación de estas lesiones en la población y obtener patrones de distribución y asociación más exactos.

\section{BIBLIOGRAFÍA}

1. Jing W, Xuan M, Lin Y, Wu L, Liu L, Zheng X, Tang W, Qiao J, Tian W. Odontogenic tumours: a retrospective study of 1642 cases in a Chinese population. J Oral Maxillofac Sur 2008 Enero; 36:20-5.

2. Luo HY, Li TJ. Odontogenic tumors: A study of 1309 cases in a Chinese population. Oral Oncol 2009 Agosto; 45: 706-711.
3. Fernandes A, Duarte E, Pimenta F, Souza L, Santos V, Mesquita R, de Aguiar M. Odontogenic tumors: a study of 340 cases in a Brazilian population. J. Oral Maxillofac Surg 2005 Noviembre;34:583-7.

4. Ochsenius G, Ortega A, Godoy L, Peñafiel C, Escobar E. Odontogenic tumors in Chile: a study of 362 cases J. Oral Maxillofac. Surg 2002 Agosto; 31:415-20.

5. Avelar R, Antunes A, Santos T, Andrade E, Dourado $E$. Odontogenic tumors: clinical and pathology study of 238 cases. Braz J Otolaryngol 2008 Octubre;74:666-73.

6. El-Gehani R, Orafi M, Elarbi M, Subhashraj K. Benign tumours of orofacial region at Benghazi, Libya: A study of 405 cases. J Cranio-Maxillo Fac Surg 2009 Febrero;37:370-5.

7. Hidalgo O, Leco M, Martínez J. Metaanalysis of the epidemiology and clinical manifestations of odontomas. Med Oral Pathol Oral Surg 2008 noviembre;13:730-4.

\section{CORRESPONDENCIA}

Carla Yamila Thiers Leal.

Teléfono: 09 - 61591833

Correo electrónico: carlathiers@gmail.com 